\title{
Single-Stage Inverter-Based Grid-Connected Photovoltaic Power Plant with Ride-Through Capability over Different Types of Grid Faults
}

\author{
Mitra Mirhosseini ${ }^{(1)}$, Josep Pou ${ }^{(1)(2)}$, and Vassilios G. Agelidis ${ }^{(1)}$ \\ ${ }^{(1)}$ Australian Energy Research Institute \& School of Electrical Engineering and Telecommunications \\ The University of New South Wales (UNSW), Sydney, NSW, 2052, Australia \\ ${ }^{(2)}$ Terrassa Industrial Electronics Group \& Department of Electronic Engineering \\ Technical University of Catalonia (UPC), Catalonia, Spain \\ Email: m.mirhosseini@student.unsw.edu.au
}

\begin{abstract}
In this paper the control of a single-stage grid-connected photovoltaic power plant (GCPPP) is developed to address the issue of inverter disconnection under various grid faults. There are three main reasons for inverter disconnection which are (i) excessive dclink voltage, (ii) excessive ac currents and (iii) loss of grid-voltage synchronization. The control of the inverter incorporates reactive power support in the case of voltage sags based on grid code requirements to ride-through the faults and support the grid voltages. Accordingly, another requirement of the grid codes is to control the reactive current injection under unbalanced voltage sags so that the voltages in the non-faulty phases do not exceed the specified limitations. All these issues are discussed in this paper for a case study of 1-MVA GCPPP using MATLAB/Simulink. The results illustrate the capability of the developed GCPPP to ridethrough different types of faults occurred on the grid side.
\end{abstract}

Index Terms-Photovoltaic systems, Large-scale systems, Power system faults, Fault-ride-through, Reactive power support.

\section{INTRODUCTION}

Nowadays, grid-connected photovoltaic power plants (GCPPPs) have received a great importance and popularity in both domestic and large-scale power generation. As the number and size of the photovoltaic (PV) power plants increase, new issues arise from both the PV power plant and grid sides. Some of these issues have been pointed out in [1]. Therefore, more investigations are required to understand the impact of GCPPPs on existing power grids and to achieve stable and secure operation.

Fault studies of GCPPPs have been widely reported in the technical literature. For instance, fault current contributions of PV power plants have been studied in [2-4]. Other research has focused on low-voltage ride through (LVRT) capability techniques [5],[6]. For unbalanced voltage sags, a method to mitigate the peak output currents of a $4.5-\mathrm{kVA}$ PV power plant in non-faulty phases is introduced in [7]. Another study in [8] presents a proportional-resonant (PR) current controller for the current limiter to assure a sinusoidal output current without overcurrent. However, in the mentioned methods, reactive power support has not been considered. For the grid-connected currentsource inverter-based PV power plants some studies have been reported in [9],[10] by considering symmetrical and asymmetrical types of faults and their impacts on the output current. However, the output current remains limited in all types of faults due to the implementation of a current source model for the inverter. For the dc side of the inverter, a study has been reported in [11] which shows the impact of various types of faults on the voltage and current of the PV array. In [12], some research has been done on a superconducting magnetic energy storage (SMES) system that is used to store energy and regulate the ac currents and the dc-link voltage during voltage sags. In [13], a method has been introduced to control the positive and negative sequences besides limiting the output current not to exceed a predefined boundary.

Considering a single-stage grid-connected inverter, no paper has proposed a comprehensive strategy for protecting both the dc and the ac sides of the inverter during unbalanced grid faults while providing reactive power support. Furthermore, there seems to be no paper that has discussed how the inverter has to be designed and modified to accommodate various types of faults and address the fault-ride-through (FRT) capability based on the grid codes [14].

The objective of this paper is to introduce some control strategies for single-stage GCPPPs that allow the inverter to remain connected under various types of grid faults based on the grid codes. PV inverter disconnection under grid faults is due to three main factors: (i) excessive dc-link voltage, (ii) excessive ac currents and (iii) loss of grid voltage synchronization, which may conflict with FRT capability. Moreover, during unbalanced conditions, the voltage in non-faulty phases should not exceed the specified limits due to reactive power injection.

The rest of the paper is organized as follows. A summary of the grid codes (GCs) for medium voltage GCPPPs is presented in Section II. A case study is introduced in Section III. In Section 
IV, the grid synchronization method used in this system is presented. The reason for inverter disconnection due to excessive ac current is assessed and a remedy is proposed in Section V. Solutions for the other reasons of inverter disconnection, i.e. excessive dc-link voltage and ac voltage exceeding in non-faulty phases, are proposed in Sections VI and VII, respectively. Finally, Section VIII summarizes the conclusions.

\section{GRID CODES REQUIREMENTS}

When connecting PV plants to medium voltage grids, it is necessary to provide them with dynamic support for the grid voltages. This dynamic support is referred to FRT technique in which the PV plant should stay connected in the case of grid faults depending on the fault time duration. They have also to provide support to the grid voltages by injecting reactive power. Considering the FRT capability, there are four major reasons for inverter disconnection during grid faults, which are the following ones: (1) excessive dc-link voltage, (ii) overcurrent at the ac side and (iii) loss of grid voltage synchronization. Moreover, in the case of asymmetrical faults, (iv) the reactive current injected must not exceed values that cause voltages to increase above $110 \%$ of the nominal value in the non-faulty phases [14].

\section{CASE STUDY}

In this paper, a 1-MVA single-stage grid-connected PV power plant is analyzed. It is modeled using MATLAB/Simulink and the system main characteristics are summarized in Table I from [15],[16]. Fig. 1 shows the model of the grid-connected PV generator, and Fig. 2 presents a basic control schematic for the transformed $d q$ current references. $i_{\text {dref }}$ and $i_{\text {qref }}$ are obtained from the dc-link voltage control and the droop control, respectively [17]. These values will be modified later in this paper according to the FRT requirements. The equation for the droop control is as follows:

$$
\begin{aligned}
& i_{\text {qref }}=d \text { roop } \times\left|d v_{a c}\right| \times I_{n}, \\
& \text { for }\left|d v_{a c}\right| \geq 10 \% \quad \& \quad \text { droop } \geq 2,
\end{aligned}
$$

in which $d v_{a c}$ is the amount of voltage drop, $I_{n}$ is the nominal current value of the inverter and droop is a constant value equal to 2. Other parameters in Fig. 1 are dc-link voltage reference $\left(V_{d c r e f}\right)$, dc-link voltage $\left(v_{d c}\right)$, line-to-line rms voltage $\left(v_{a c}\right)$ and nominal rms voltage $\left(V_{n}\right) \cdot i_{\text {qmin }}$ and $i_{\text {qmax }}$ are the lower and upper reactive current limitations, respectively, which are equal to $I_{n}$.

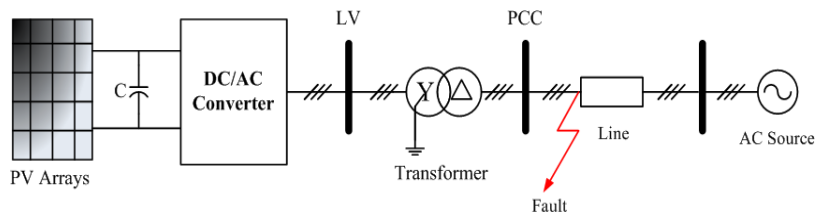

Fig. 1. Schematic of a single-stage GCPPP.
TABLE I. CASE Study System Characteristics

\begin{tabular}{|c|c|c|c|}
\hline \multicolumn{2}{|c|}{$\begin{array}{c}\text { PV Module Characteristics in } \\
\text { Standard Test Conditions } \\
\text { (STC) }\end{array}$} & \multicolumn{2}{c|}{ PV Inverter Characteristics } \\
\hline $\begin{array}{c}\text { Optimum } \\
\text { Operating } \\
\text { Voltage, } V_{m p p}\end{array}$ & $35.6 \mathrm{~V}$ & $\begin{array}{c}\text { Max. dc Power } \\
\text { (at cos } \varphi=1)\end{array}$ & $1133 \mathrm{~kW}$ \\
\hline $\begin{array}{c}\text { Optimum } \\
\text { Operating } \\
\text { Current, } I_{m p p}\end{array}$ & $8.29 \mathrm{~A}$ & $\begin{array}{c}\text { Max. dc Input } \\
\text { Voltage }\end{array}$ & $1000 \mathrm{~V}$ \\
\hline $\begin{array}{c}\text { Open Circuit } \\
\text { Voltage, } V_{o c}\end{array}$ & $44.3 \mathrm{~V}$ & $\begin{array}{c}\text { Max. Output } \\
\text { Current }\end{array}$ & $31.8 \mathrm{~A}$ \\
\hline $\begin{array}{c}\text { Short Circuit } \\
\text { Current, } I_{s c}\end{array}$ & $8.74 \mathrm{~A}$ & $\begin{array}{c}\text { Rated ac } \\
\text { Voltage }\end{array}$ & $20 \mathrm{kV}$ \\
\hline $\begin{array}{c}\text { Number of } \\
\text { Parallel Modules }\end{array}$ & 155 & $\begin{array}{c}\text { Rated ac Power } \\
\text { (at STC) }\end{array}$ & $1100 \mathrm{kVA}$ \\
\hline $\begin{array}{c}\text { Number of Series } \\
\text { Modules }\end{array}$ & 22 & Transformer & $\begin{array}{c}1.2 \mathrm{MVA} \\
\text { Dyn } 11 \\
50 \mathrm{~Hz}\end{array}$ \\
\hline
\end{tabular}

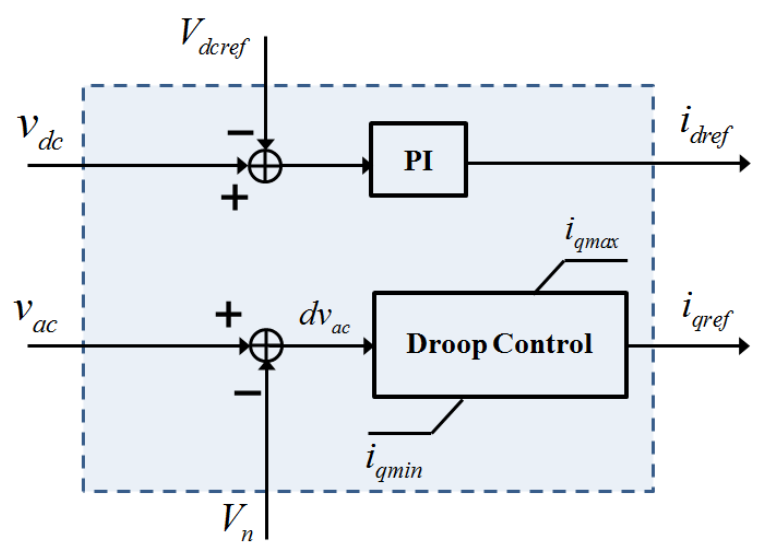

Fig. 2. Basic schematic of the transformed $d q$ reference currents.

In [18], concerning the FRT capability, the reasons for inverter disconnection are described for difference voltage sags conditions based on the German GCs [14].

\section{GRID VOLTAGE SYNCHRONIZATION}

In grid-connected inverters, one important issue is the voltage phase angle detection. The phase-locked-loop (PLL) method is one of the key technologies extensively used, which is based on a synchronous reference frame PLL (SRF-PLL) [19]. However, it has been demonstrated that the conventional PLL configuration does not perform well under asymmetrical faults and consequently leads to inverter being disconnected from the grid [18]. In this paper, an alternative PLL method is used based on the moving average filters (MAFs) introduced in [20] and implemented in [18]. 


\section{EXCESSIVE AC CURRENT}

Commercial grid-connected inverters have a maximum ac current value specified; if the currents exceed this limitation the inverter is disconnected from the grid. Under a grid voltage sag condition, the $d$-component of the current (in a synchronous reference frame) starts to increase due to the voltage drop at the grid side. Consequently, the grid currents increase, which may lead the overcurrent protection actuate and force the inverter to be disconnected from the grid.

For this case study, according to the characteristics of the PV modules and the number of units connected in series and parallel, the maximum power injected under standard test conditions (STC) is $1.006 \mathrm{MW}$. This power gives the rated current of 1400A (1979A peak value) at the low voltage side of the transformer. According to the datasheet of the inverter, the maximum acceptable output current at the low voltage side of the transformer is 1532A (2167A peak value). However, for a $40 \%$ three-phase-to-ground voltage sag at the point of common coupling (PCC), the peak value of the output current exceeds the limits in the example shown in Fig. 3, which leads to inverter disconnection.

Therefore, the references provided to the current controllers of the inverter have to be limited based on the maximum acceptable currents. This would allow the inverter to keep on operating during some voltage sag processes without overpassing the current protection limits and hence forcing it to be disconnected from the grid. Moreover, based on the requirements of FRT capability of injecting reactive current under fault conditions, the current limiter should limit the active current reference if necessary to give sufficient room for the reactive current injection. The reactive current is assigned according to the droop control (1). The modified reference currents after passing through the current limiter are assigned as $i_{\text {dref }}^{\prime}$ and $i_{\text {qref }}^{\prime}$.

If the generated power in the dc-side of the GCPPP is more than the injected power into the electrical grid, the dc-link capacitor voltage will start to increase. Consequently, in a singlestage PV power plant, as the dc-link voltage rises the operating point on the I-V curve moves towards the open-circuit voltage point, which leads the PV current to decrease, as shown in Fig. 4. Therefore, the power generated by the PV power plant is reduced because the operating point is taken away from the maximum power point (MPP) and therefore less active current is required in the ac-side.

Finally, the operating point reaches new values in which the input current is lower and the dc-link voltage higher than the values prior to the voltage sag event, as shown in Fig. 5(a). Thus, this GCPPP is self-protected because the generated power is reduced when the dc-link voltage increases under fault conditions. It should be mentioned that the inverter have to be able to withstand such an increase on the dc-link voltage which will be addressed in Section VI. In Fig. 5(b), by the implementation of the current limiter and injection of the reactive current during the voltage sag, the active current is reduced. It is worth mentioning that in two-stage grid-connected inverters, the MPP tracking is performed by the $\mathrm{dc} / \mathrm{dc}$ converter and the dc-link voltage is controlled by the inverter. Therefore, the GCPPP is not self-protected during the fault conditions because there is no reduction in the generated power. A specific control action should be performed to reduce the generated power in such a configuration.

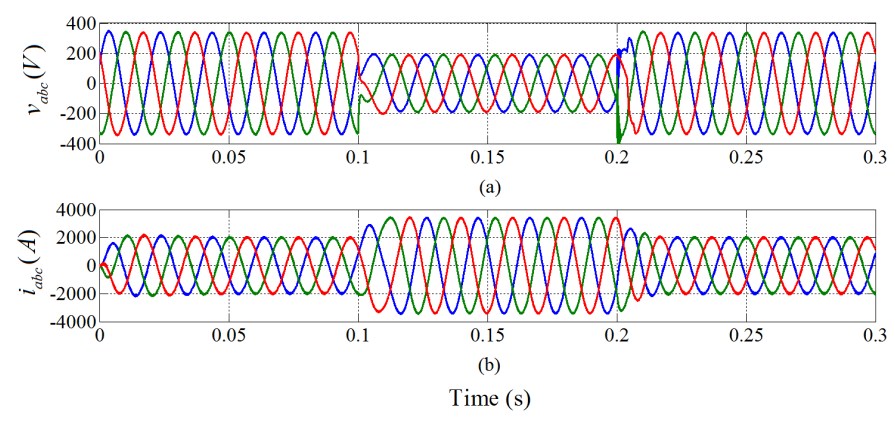

Fig. 3. Grid (a) voltages at the PV power plant terminal under threephase $40 \%$ voltage sag and (b) grid currents.

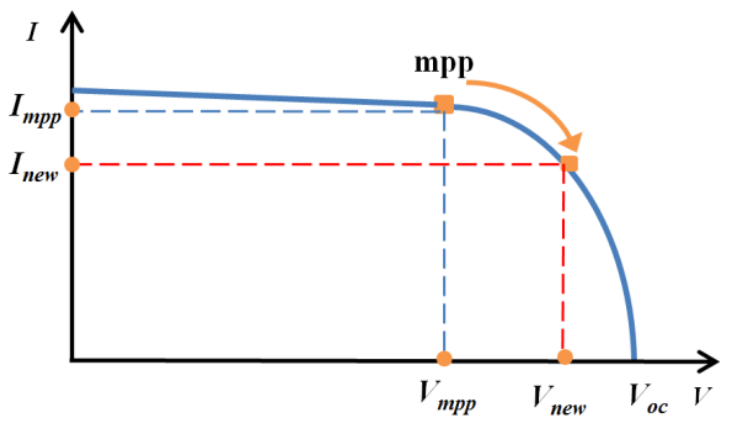

Fig. 4. Change in the operating point of the PV array under a voltage sag condition.

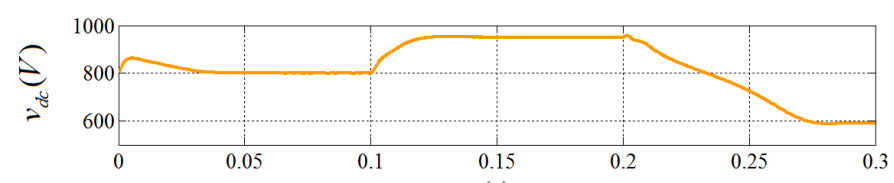

(a)

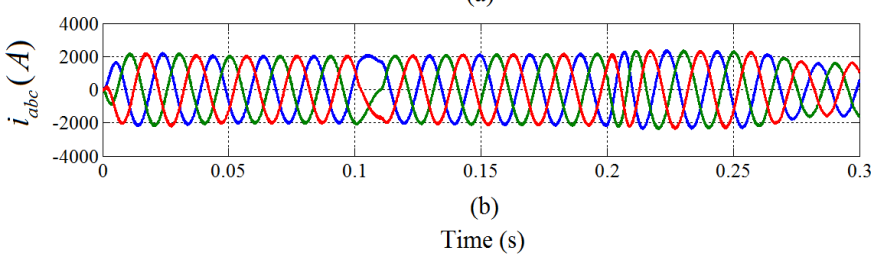

Fig. 5. Application of a current limiter: (a) dc-link voltage and (b) grid currents under a three-phase $40 \%$ voltage sag. 


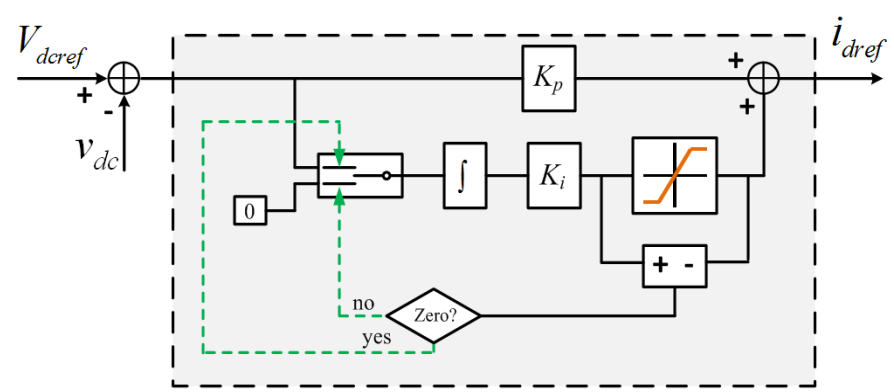

Fig. 6. PI controller with an anti-windup technique.

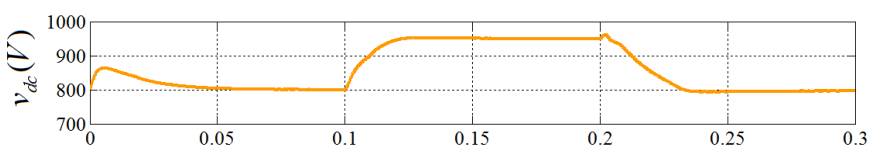

(a)

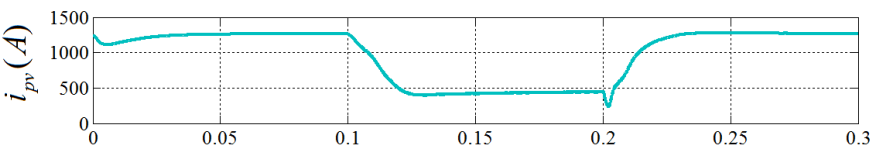

(b)

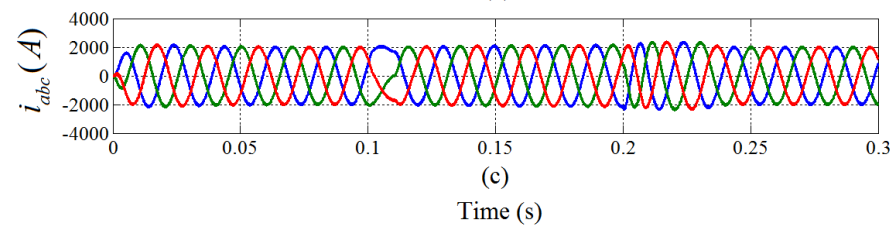

Fig. 7. Application of an anti-windup strategy to the PI controller: (a) dc-link voltage, (b) PV current, and (c) grid currents.

A problem that may arise because of the deviation of the MMP during the voltage sag is that after fault removal the dclink voltage and the ac currents may take a long time to reach the pre-fault values, as shown in Fig. 5. The reason for this is the accumulation of error in the integral part of the proportionalintegral (PI) dc-link voltage controller. The existing dc-link voltage error keeps the PI output increasing prior the current limiter, and therefore excessive control action may be accumulated during the voltage sag process. To overcome this issue, an anti-windup technique is proposed to stop the PI controller to accumulate excessive control action when it exceeds a specified value [21]. A schematic of anti-windup technique is shown in Fig. 6. The results when applying the anti-windup technique are depicted in Fig. 7. One can observe that all the magnitudes reach the pre-fault values very fast after fault removal at $t=0.2 \mathrm{~s}$.

From another perspective, the rms value of the voltage contains even-order harmonics under unbalanced grid voltage conditions which makes the rms voltage drop $\left(d v_{a c}\right)$ distorted. Consider two-phase-to-ground (2LG) fault with 35\% voltage drop in faulty phases. $d v_{a c}$ is as shown in Fig. 8(a). The droop control defines to inject reactive current when the voltage drop is more than $10 \%$ of the nominal voltage. Therefore, based on the amount of voltage drop, the droop control may be activated and deactivated continuously. This distorts the $i_{\text {qref }}^{\prime}$ and also $i_{\text {dref }}^{\prime}$ after passing through the current limiter, as depicted in Fig. 8. Distorted reference currents produce the distorted ac currents shown in Fig. 9, which may lead to overcurrent in some cases.

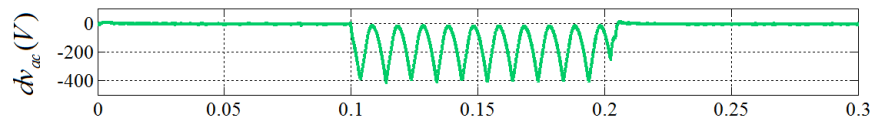

(a)

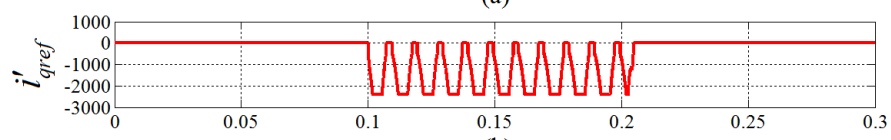

(b)

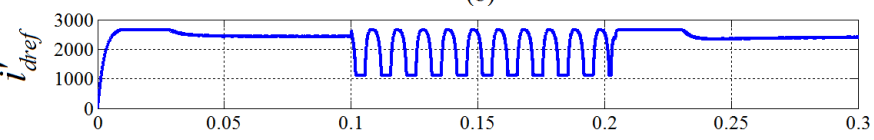

(c)

Time (s)

Fig. 8. Unbalanced grid voltag sag due to a $2 \mathrm{LG}$ fault: (a) voltage drop, (b) reactive current reference, and (c) active current reference.

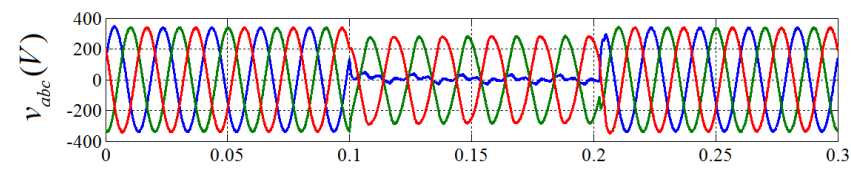

(a)

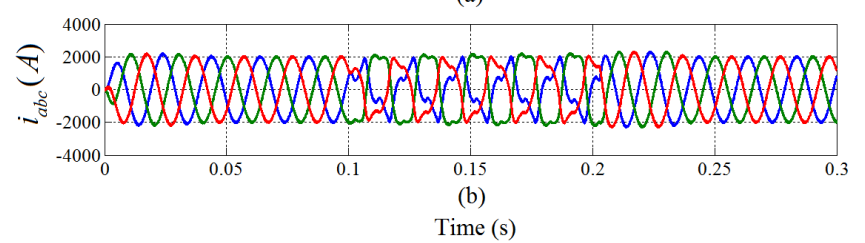

Fig. 9. (a) PV terminal voltages under 2LG fault and (b) output currents.
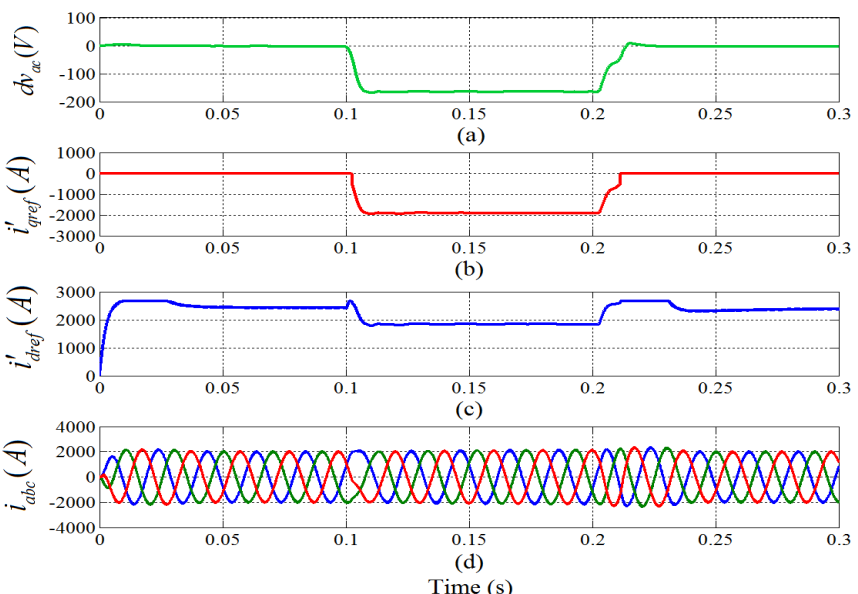

Fig. 10. Including a MAF in the control: (a) voltage drop, (b) reactive reference current, (c) active reference current, and (d) output currents. 
To overcome this problem a new method is introduced which is described in [17]. This method includes a MAF technique which is used for the voltage drop. This filter removes the second-order harmonics of the rms voltage and as a result the output current will be sinusoidal. The improved results are depicted in Fig. 10.

This method is effective when the PV power plant control loops are designed to perform in the synchronous reference frame. Another alternative is to employ natural control frame ( $a b c$-frame) by using a droop control for each phase as well as individual PR controllers in the current control loops. There would be no need for applying MAFs or any other filtering strategies in this case. The authors will focus on this method in their future studies.

\section{EXCESSIVE DC-LINK VOLTAGE}

As it was shown in Section $\mathrm{V}$, the dc-link voltage starts to rise during the voltage sag to reach a new steady-state point. Therefore, the inverter has to withstand the worst case of the dclink voltage, which is produced when the voltage provided by the PV modules reaches the open-circuit value $\left(V_{o c}\right)$ under STC. Hence, the number of PV modules connected in series $\left(n_{s}\right)$ has to be limited during the design of the GCPPPs so that the dc-link voltage is never higher than the maximum acceptable value of the inverter $\left(V_{d c-\max }\right)$ as follows:

$$
n_{s} \times V_{o c} \leq V_{d c-\max } \Rightarrow n_{s} \leq \frac{V_{d c-\max }}{V_{o c}} .
$$

This maximum voltage restriction is represented in Fig. 11.

According to the data provided in Table I, the total voltage of the series modules in the worst case, i.e. open circuit and maximum solar radiation, is $974.6 \mathrm{~V}$. This is less than the maximum voltage that the inverter can tolerate at the dc side $(1000 \mathrm{~V})$.

\section{Voltage ExCEEDING IN NON-FAUlty PhaseS}

Under unbalanced fault conditions, reactive currents are injected into the electricity grid according to the value of voltage drop. Because the reactive currents are balanced, there is a possibility of voltage exceeding in the non-faulty phases. For a $415 \mathrm{~V}$ rms voltage the maximum acceptable voltage peak value (i.e. $110 \%$ of nominal value) is $372.73 \mathrm{~V}$. In Fig. 12, the grid voltages are magnified to show the impact of reactive current injection in the grid voltages during a two-phase-to-ground fault. As it can be observed, the voltage in the non-faulty phase is excessively increased due to the reactive current injection. To overcome this problem, the maximum value of the injected reactive current has to be limited. In the case of normal control approach, the lower boundary of the reactive current is the nominal current of the PV generator. However, to avoid grid overvoltages, the lower boundary should be reduced to limit the amount of injected reactive current. For this purpose, a PI controller is employed to regulate the maximum value of the phase voltage. As shown in Fig. 13, the amount of reactive current injection is limited, and the peak value of the voltage is reduced from $383 \mathrm{~V}$ to $372 \mathrm{~V}$. The whole process is shown in Fig. 14 in which $v_{\max }$ is the maximum instantaneous voltage of the three phases. However, as it can be seen from Fig. 13, the reaction of reactive current reduction is faster than its effect on the voltage value. If the PI controller is selected to be slower it will take a longer time to achieve a voltage reduction, while if it is selected to be faster the reactive current tends to zero earlier. The reason is the slow nature of the voltage profile that can be affected by the reactive current change, which is not desirable under fault conditions.

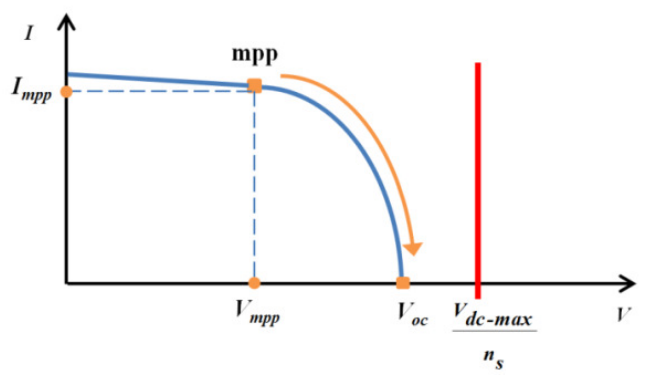

Fig. 11. The maximum dc-link voltage has to be higher that the PV voltage in open circuit $\left(V_{o c}\right)$.

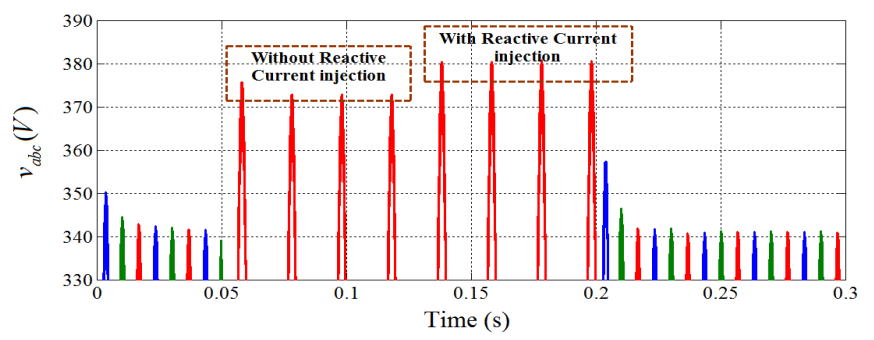

Fig. 12. Grid voltage exceeding in the non-faulty phase due to symmetrical reactive current injection.
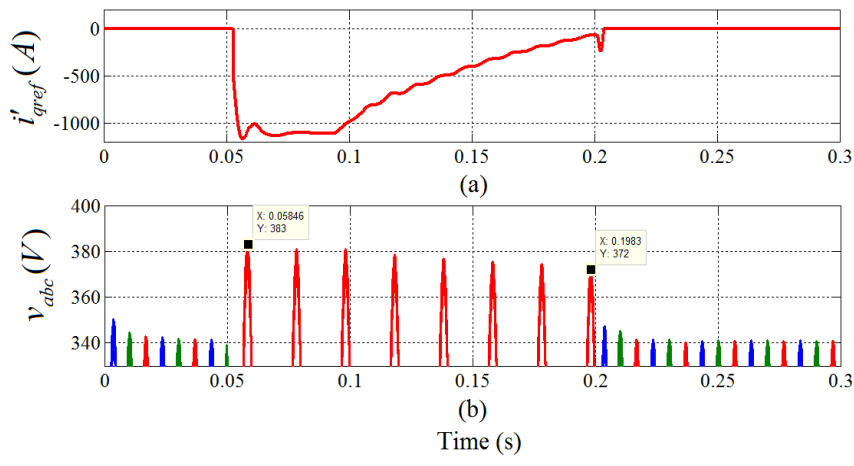

Fig. 13. Application of a controller to limit the rise of the voltage in the non-faulty phase: (a) reactive current reference and (b) grid voltages. 


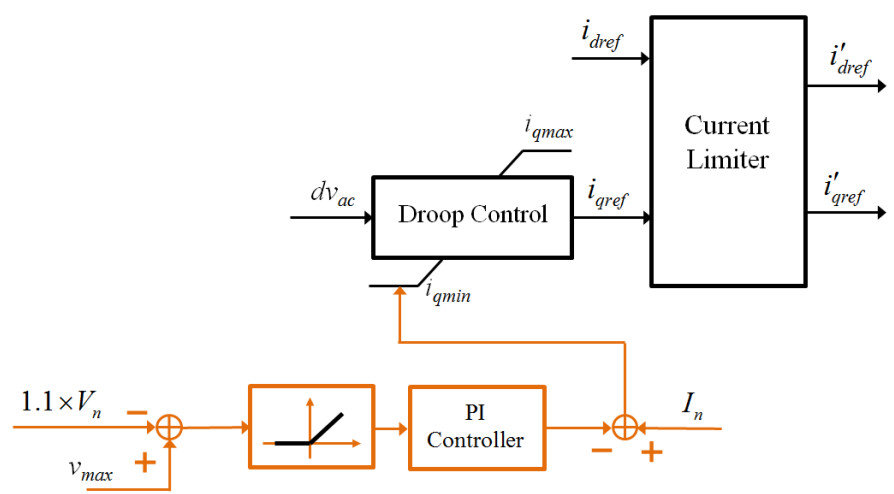

Fig. 14. Control diagram limiting the reactive current lower boundary.

Therefore, this is not actually a comprehensive method as the time period to ride-through the fault is very short (in German GCs the maximum time to ride-through for $100 \%$ voltage drop is only $150 \mathrm{~ms})$. Therefore, a more effective remedy to be compatible with FRT time frame which is based on supplying the unbalanced voltage with unbalanced reactive currents is recommended. Such development is to be reported soon but is beyond the scope of this paper.

\section{CONCLUSION}

In this paper, the issues of GCPPP under fault conditions are addressed for single-stage grid-connected inverters. For this purpose, some extra modifications need to be applied to make the PV power plant ride-through compatible with any type of faults according to the grid codes. These modifications include applying current limiters, filtering techniques to remove the distortion from the reference currents, and developing a control strategy to control the lower boundary of the generated reactive current.

\section{REFERENCES}

[1] F. Katiraei and J. R. Aguero, "Solar PV integration challenges," IEEE Power Energy Mag., vol. 9, pp. 62-71, Apr. 2011.

[2] R. J. Bravo, R. Yinger, S. Robles, and W. Tamae, "Solar PV inverter testing for model validation," in Proc. IEEE Power and Energy Society General Meeting, 2011, pp. 1-7.

[3] S. Phuttapatimok, A. Sangswang, M. Seapan, D. Chenvidhya, and K. Kirtikara, "Evaluation of fault contribution in the presence of PV gridconnected systems," in Proc. 33rd IEEE Photovoltaic Specialists Conference (PVSC), 2008, pp. 1-5.

[4] T. S. Sidhu and D. Bejmert, "Short-circuit current contribution from large scale PV power plant in the context of distribution power system protection performance," in Proc. IET Conference on Renewable Power Generation 2011, pp. 1-6.

[5] A. Marinopoulos, F. Papandrea, M. Reza, S. Norrga, F. Spertino, and R. Napoli, "Grid integration aspects of large solar PV installations: LVRT capability and reactive power/voltage support requirements," in Proc. IEEE Trondheim PowerTech, 2011, pp. 1-8.
[6] G. M. S. Islam, A. Al-Durra, S. M. Muyeen, and J. Tamura, "Low voltage ride through capability enhancement of grid connected large scale photovoltaic system," in Proc. IECON 2011 - 37th Annual Conference on IEEE Industrial Electronics Society, 2011, pp. 884-889.

[7] J. Miret, M. Castilla, A. Camacho, L.G. Vicuna, and J. Matas, "Control scheme for photovoltaic three-phase inverters to minimize peak currents during unbalanced grid-voltage sags," IEEE Trans. Power Electron., vol. 27, pp. 4262-4271, 2012.

[8] G. M. S. Azevedo, P. Rodriguez, M. C. Cavalcanti, G. Vazquez, and F. A. S. Neves, "New control strategy to allow the photovoltaic systems operation under grid faults," in Proc. Brazilian Power Electronics Conference (COBEP), 2009, pp. 196-201.

[9] P. P. Dash and M. Kazerani, "Dynamic modeling and performance analysis of a grid-connected current-source inverter-based photovoltaic system," IEEE Trans. Sustain. Energy, vol. 2, pp. 443-450, 2011.

[10] A. Yazdani, A. R. Di Fazio, H. Ghoddami, M. Russo, M. Kazerani, J. Jatskevich, K. Strunz, S. Leva, and J. A. Martinez, "Modeling guidelines and a benchmark for power system simulation studies of three-phase single-stage photovoltaic systems," IEEE Trans. Power Del., vol. 26, pp. 1247-1264, 2011.

[11] H. C. Seo, C. H. Kim, Y. M. Yoon, and C. S. Jung, "Dynamics of gridconnected photovoltaic system at fault conditions," in Proc. Transmission \& Distribution Conference \& Exposition: Asia and Pacific, 2009, pp. 1-4.

[12] S. T. Kim, B. K. Kang, S. H. Bae, and J. W. Park, "Application of SMES and grid code compliance to wind/photovoltaic generation system," IEEE Trans. Appl. Supercond., vol. 23, pp. 5000804-5000804, 2013.

[13] P. Rodriguez, A. Luna, J. R. Hermoso, I. Etxeberria-Otadui, R. Teodorescu, and F. Blaabjerg, "Current control method for distributed generation power generation plants under grid fault conditions," in Proc. 37th Annual Conference on IEEE Industrial Electronics Society (IECON) , 2011, pp. 1262-1269.

[14] E. Troester, "New german grid codes for connecting PV systems to the medium voltage power grid," presented at the International Conference on Concentrating Photovoltaic Power Plant, Germany, 2009.

[15] SMA Solar Technology. SUNNY CENTRAL 1000MV / 1250MV $1600 \mathrm{MV}$ for direct medium-voltage feed-in. (July 2013). [Online]. Available: http://www.sma.de/en/products/central-inverters/sunny-central1000mv-1250mv-1600mv.html\#Technical-Data-14803

[16] SUNTECH POWER. 295 Watt polycrystalline solar module. (July 2013). [Online]. Available: http://www.suntechpower.com/images/stories/pdf/datasheets/en-GB/STP 285305 Ve(poly)_EN_web.pdf

[17] M. Mirhosseini, J. Pou, and V. G. Agelidis, "Current improvement of a grid-connected photovoltaic system under unbalanced voltage conditions," in Proc. IEEE Annual International Energy Conversion Congress and Exhibition (ECCE ASIA), Australia, 2013, pp. 66-72.

[18] M. Mirhosseini and V. G. Agelidis, "Performance of large-scale gridconnected photovoltaic system under various fault conditions," in Proc. IEEE International Conference on Industrial Technology (ICIT), 2013, pp. 1775-1780.

[19] L. Shi and M. L. Crow, "Adaptive quadrant filter based phase locked loop system," in Proc. North American Power Symposium (NAPS), 2010, pp. 17.

[20] E. Robles, S. Ceballos, J. Pou, J. L. Martin, J. Zaragoza, and P. Ibanez, "Variable-frequency grid-sequence detector based on a quasi-ideal lowpass filter stage and a phase-locked loop," IEEE Trans. Power Electron., vol. 25, pp. 2552-2563, Oct. 2010.

[21] J. Espina, A. Arias, J. Balcells, and C. Ortega, "Speed anti-windup PI strategies review for field oriented control of permanent magnet synchronous machines," in Proc. Compatibility and Power Electronics, 2009, pp. 279-285. 